\title{
Genetics of pre-eclampsia and the weight of babies at birth - clinical and genetic studies in Sri Lanka
}

\author{
V H W Dissanayake ${ }^{1}$ \\ (Index words: pre-eclampsia, birthweight, MTHFR, F2, F5, EGF, AGT, TGFA)
}

\section{Background}

Pre-eclampsia is the de novo occurrence of hypertension and proteinuria in pregnancy in an otherwise normal woman [1]. Recent advances in genetics has resulted in a surge of investigations into genetic factors underlying pre-eclampsia [2,3]. These studies have been conducted mainly among western Europeans and Japanese. The investigations reviewed in this paper were undertaken in a genetically distinct South Asian Sinhalese population in Sri Lanka.

Complex disorders such as pre-eclampsia are caused by low penetrant, high frequency genetic variations in several genes interacting with each other and the environment [2]. As a result the genetic basis of preeclampsia is not immediately clear [4]. Genetic traces of pre-eclampsia could, however, be unmasked through epidemiological studies, twin studies, and pedigree analysis using special techniques [5]. Epidemiological studies show that both maternal and paternal genes, acting through the foetus, may contribute to the development of pre-eclampsia [6]. Twin studies confirm this [7], and pedigree studies report that the pattern of inheritance of pre-eclampsia is consistent with an autosomal dominant pattern with reduced penetrance [4].

Two strategies can be used to identify genes causing pre-eclampsia - linkage studies and candidate gene disease association studies [5]. Linkage studies involve recruiting families with multiple affected members, and then identifying regions of the genome that are co-inherited along with pre-eclampsia within the family using molecular genetic tests to analyse their DNA. There have been several genome wide linkage screens for pre-eclampsia. Genetic loci harbouring a gene or genes involved in preeclampsia are as follows: 4q, 2p13, 2p25, 9p13 [2]. However there is little overlap between the loci identified in different populations in these screens, possibly because of genetic differences in populations. This highlights the importance of carrying out genetic studies in different parts of the world. However, it is not possible to carry out linkage studies in many parts of the world because medical and geneological records that are necessary to ascertain such families are not widely available.
Candidate gene disease association studies, on the other hand, can be performed anywhere. These studies involve prospectively recruiting cases and controls from a population, selecting a candidate gene and the genetic markers in or near the candidate gene, and then determining whether the marker is found more commonly in cases than in controls using molecular genetic tests. This was the approach we selected for our studies of the genetics of pre-eclampsia in Sri Lanka.

Genetic markers are genetic variations. There are various types of genetic markers. The preferred markers for case control studies are single nucleotide polymorphisms or single base changes in the genome [8]. There are two ways of selecting candidate genes. First, positional candidates, i.e. genes located in genomic regions identified by genome wide linkage screens. Second, functional candidates, i.e. genes that code for any protein that is involved in the pathophysiological pathway of preeclampsia [2].

Candidate gene disease association studies have been the popular method used to study genetics of preeclampsia. Many candidate genes across the genome have been studied using this approach [3]. However, a limitation of these studies has been that the results in one population have not been consistently reproducible in other populations - an important consideration in true associations. There are four main reasons for this. Firstly, there are issues relating to the definition of pre-eclampsia because blood pressure and urinary protein are variable measurements. Establishing the diagnosis involves passing pre-defined thresholds or cut offs on which there has been some controversy $[9,10]$ prior to the adoption of new guidelines by the International Society for the Study of Hypertension in 2001 [1]. Secondly, researchers fail to rigorously phenotype cases. Some medical conditions and obstetric states are known to increase the risk of development of pre-eclampsia. Therefore, careful phenotyping is necessary when recruiting cases and controls for these studies. Thirdly, there is the possibility of population admixture, i.e. the presence of different inbreeding subpopulations within cases and controls. This could happen when careful attention is not paid to determination of the ethnicity of recruits and when cases and controls are not matched for ethnicity. Finally, most

${ }^{1}$ Human Genetics Unit, Faculty of Medicine, University of Colombo, Sri Lanka.

Correspondence: VHWD, e-mail <vajirahwd@hgucolombo.org>. Received 6 August 2008 and revised version accepted 10 January 2009. Competing interests: none declared. 
studies do not have sufficient numbers of cases and controls, making them underpowered to detect any genetic effect [11]. We were mindful of these problems when we began our studies into the genetics of pre-eclampsia in Sri Lanka.

\section{Genetic studies in Sri Lanka}

By applying a strict definition for pre-eclampsia and rigorous exclusion criteria, we recruited a group of women who were carefully phenotyped for pre-eclampsia. The issue of population admixture was addressed as far as possible [12,13,14]. A sample size of 180 cases and 180 controls was calculated to have adequate power to detect a doubling of risk of pre-eclampsia when the susceptibility allele of the genetic marker that increased pre-eclampsia risk ranged from 0.1 to 0.5 , using a dominant model, at a significance level of $\mathrm{p}=0.05$ with $80 \%$ power.

\section{Candidate gene studies}

We selected candidate genes after a review of scientific writing. They were methylenetetrahydrofolate reductase (MTHFR), prothrombin (Factor II, F2), factor V (F5), angiotensinogen (AGT), transforming growth factor alpha (TGFA), epidermal growth factor (EGF). We selected both positional (TGFA and EGF) and functional (MTHFR, F2, F5, TGFA, EGF, AGT) candidates. We selected genes that have been studied previously (MTHFR, F2, F5, AGT) and genes that have not been studied previously (TGFA and EGF). Next we selected the genetic markers. They were all single nucleotide polymorphisms. They were as follows: MTHFR 677C $>$ T, 1298A $>$ C, 1317T $>$ C, 1793G $>$ A; F2 20210G $>$ A; F51691G $>$ A, 4070A $>$ G; TGFA 3822G $>$ A; 3827T $>$ C, 3851T $>$ C; EGF 61G $>$ A, 2566G $>$ A;AGT 174T $>$ M, $235 \mathrm{M}>\mathrm{T}, 11535 \mathrm{C}>\mathrm{A}$.

Results of genotyping the population based DNA collection for the genetic markers showed that MTHFR 1317T >C and F2 20210G > A were not polymorphic in our population $[15,16]$. The susceptibility allele frequencies of the Tamils and Moors were similar to that of the Sinhalese. Therefore, any unsuspected population admixture would not affect the results of our candidate gene disease association studies. The results of our candidate gene disease association studies showed that the $\mathrm{G}$ allele of the $2566 \mathrm{G}>\mathrm{A}$ polymorphisms in the EGF gene increased the risk of pre-eclampsia. Women who were homozygous for the allele had almost doubling of risk [OR $(95 \%$ CI $)=1.87(1.05-3.31)$ [17].

\section{Genetics of birth weight}

The collection of extensive clinical and genetic data opens up opportunities for innovative analyses. We decided to explore the contribution of genetic variants to the weight of babies at birth. The birthweight of babies born to women with pre-eclampsia is usually low. We found that the birthweight of babies born to women who had uncomplicated pregnancies was associated with the mother's epidermal growth factor genotype [15].

The maternal-fetal interface in pregnancy is unique. It is the only site in an organism where genes from the mother and genes from the father, acting through the foetus, interact. To study this interaction the genetic make up of the father, the mother and the foetus or the baby the so called parent-child trios - has to be investigated $[17,18]$. When we genotyped these trios and analysed the data we found that chromosomes that contained the $61 \mathrm{G}$ and 2566A alleles of the EGF gene or the 61G/2566A haplotype which is associated with lower birthweight, was preferentially transmitted by heterozygous parents to their growth restricted babies [15].

\section{Conclusions}

Our findings may have important implications when taken in the context of the Barker hypothesis [19]. The premise of the Barker hypothesis is that adult onset disorders have origins in foetal life. Birthweight, which is the surrogate marker for development in foetal life, is an important predictor of health. Low birthweight is related to an increased risk of diseases in adult life, including cardiovascular disease, hypertension and diabetes. It raises the question, therefore, whether the epidermal growth factor gene could be the link between low birthweight and adult onset disorders. If it turns out to be so, epidermal growth factor would be a potential molecular target for targeted therapy to prevent these adult onset disorders.

\section{Acknowledgements}

I wish to acknowledge my supervisors, coinvestigators, obstetricians at the De Soysa Hospital for Women and the Castle Street Hospital for women (2001 2003) and their staff, the women who took part in these investigations, Prof. Rohan Jayasekara for critical appraisal of the manuscript, and funding from the President's Fund of Sri Lanka, University of Colombo, University of Nottingham, SLMA Glaxo-Welcome Research Award 2001, National Science Foundation Grant No: 2004/SIDA/BT/ 01 and IRQUE project research grants, Faculty of Medicine, Colombo 2006.

\section{References}

1. Brown MA, Lindheimer MD, de Swiet M, et al. The classification and diagnosis of the hypertensive disorders of pregnancy: statement from the International Society for the Study of Hypertension in Pregnancy (ISSHP). Hypertension in Pregnancy 2001; 20: IX-XIV.

2. Chappell S, Morgan L. Searching for genetic clues to the causes of pre-eclampsia. Clinical Science 2006; 110: 443-58. 
3. Lachmeijer AM, Dekker GA, Pals G, et al. Searching for pre-eclampsia genes: the current position. European Journal of Obstetrics and Gynaecology and Reproductive Biology 2002; 105: 94-113.

4. Arngrimsson R, Bjornsson H, Geirsson RT. Analysis of different inheritance patterns in pre-eclampsia/eclampsia syndrome. Hypertension in Pregnancy 1995; 14: 27-38.

5. Lander ES, Schork NJ. Genetic dissection of complex traits. Science 1994; 265: 2037-48.

6. Esplin MS, Fausett MB, Fraser A, et al. Paternal and maternal components of the predisposition to pre-eclampsia. New England Journal of Medicine 2001; 344: 867-72.

7. Salone Ros H, Lichtenstein P, Lipworth L, Cnattingius S. Genetic effects on the liability of developing pre-eclampsia and gestational hypertension. American Journal of Medical Genetics 2000; 91: 256-60.

8. Gray IC, Campbell DA, Spurr NK. Single nucleotide polymorphisms as tools in human genetics. Human Molecular Genetics 2000; 9: 2403-8.

9. Redman CW, Jefferies M. Revised definition of preeclampsia. Lancet 1988; 1: 809-12.

10. Davey DA, MacGillivray I. The classification and definition of the hypertensive disorders of pregnancy. American Journal of Obstetrics and Gynaecology 1988; 158: 892-8.

11. Lalouel JM, Rohrwasser A. Power and replication in casecontrol studies. American Journal of Hypertension 2002; 15: $201-5$

12. Dissanayake VHW, Morgan L, Broughton Pipkin F, et al. The urine protein heat coagulation test - a useful screening test for proteinuria in pregnancy in developing countries: a method validation study. British Journal of Obstetrics and Gynaecology 2004; 111: 491-4.

13. Dissanayake VHW, Samarasinghe D, Morgan L, et al. An analysis of the reasons for non-participation of subjects referred for recruitment for the Inherited Factors in Preeclampsia study (IFPE Study). Sri Lanka Journal of Obstetrics and Gynaecology 2004; 26: 28-9.

14. Dissanayake VHW, Samarasinghe HD, Morgan L, et al. Morbidity and mortality associated with pre-eclampsia at two tertiary care hospitals in Sri Lanka. Journal of Obstetrics and Gynaecology Research 2007; 33: 56-62.

15. Dissanayake VHW, Tower C, Broderick A, et al. Polymorphism in the epidermal growth factor gene is associated with birthweight in Sinhalese and white western Europeans. Molecular Human Reproduction 2007; 13: 425-9.

16. Dissanayake VHW, Jayasekara RW, Seneviratne HR, et al. A study of three candidate genes for pre-eclampsia in a Sinhalese population from Sri Lanka. Journal of Obstetrics and Gynaecology Research. In press.

17. Mitchell LE. Differentiating between fetal and maternal genotypic effects, using the transmission test for linkage disequilibrium. American Journal of Human Genetics 1997; 60: $1006-7$

18. Spielman RS, McGinnis RE, Ewens WJ. Transmission test for linkage disequilibrium: the insulin gene region and insulindependent diabetes mellitus (IDDM). American Journal of Human Genetics 1993; 52: 506-16.

19. Barker DJ, Winter PD, Osmond C, et al. Weight in infancy and death from ischaemic heart disease. Lancet 1989; 2: 577-80. 